Original Article

\title{
The effects of action observation gait training on the static balance and walking ability of stroke patients
}

\author{
Eun Cho Park, MS, PT ${ }^{1)}$, Gak Hwangbo, PhD, $\mathrm{PT}^{1)^{*}}$ \\ 1) Department of Physical Therapy, College of Rehabilitation Science, Daegu University: 15 Jillyang, \\ Gyeongsan-si, Kyeongbuk 712-714, Republic of Korea
}

\begin{abstract}
Purpose] The purpose of the study was to investigate the effects of action observation training on the static balance and walking ability of patients who had suffered a stroke. [Subjects] Forty patients with hemiplegia resulting from a stroke were divided into an action observation gait training group (AOGT group, $\mathrm{n}=20$ ) and a general gait training group (GGT group, $\mathrm{n}=20$ ). [Methods] The AOGT group watched a training video on flatland gait, slope gait, and stair gait. The GGT group watched a video on nature. Both groups watched their respective video for 10 minutes and then had gait training for 20 minutes per day, five times per week, for eight weeks. [Results] The static balance and gait ability of both groups significantly improved. Although there were significant differences between the groups, the AOTG group showed greater improvements in sway speed, limit of stability, and gait ability. [Conclusion] We recommend action observation training over general gait training for patients with hemiplegia. Action observational training had a positive effect on static balance and gait ability in stroke patients' static balance and gait ability. Further research is needed to generalize the results of this study.

Key words: Hemiplegia, Action observation training, Gait
\end{abstract}

(This article was submitted Jun. 23, 2014, and was accepted Aug. 24, 2014)

\section{INTRODUCTION}

Independent walking is an ultimate treatment goal of stroke patients and an important indicator for evaluating the recovery of motor skills ${ }^{1)}$. Methods of improving walking ability in stroke patients include gait training using functional electronic stimulation (FES) intended to improve walking ability through electrical stimulation of paralyzed muscles ${ }^{2)}$, progressive resistive exercises that improve walking ability by gradually increasing the amount of resistance to the lower extremities $^{3)}$, virtual reality systems that create situations that are similar to reality to make subjects feel as if they are being trained in such situations ${ }^{4)}$ and task-oriented training for repeated practices of walking and stair walking5); the utility of these methods has been verified by many previous researchers ${ }^{6}$. Gait training programs focus on improving motor skills through activation of the brain region using direct/indirect stimulation of the brain including cognitive treatments. Methods of activating the plasticity of the brain region include cognitive rehabilitation training programs utilizing motor nerves and sensory nerve stimulation ${ }^{7)}$. Among them, action observation training, which improves functional movements by stimulating the nerve cells in the

*Corresponding author. Gak Hwangbo (E-mail: hbgak@ daegu.ac.kr)

(C2015 The Society of Physical Therapy Science. Published by IPEC Inc. This is an open-access article distributed under the terms of the Creative Commons Attribution Non-Commercial No Derivatives (by-ncnd) License $<$ http://creativecommons.org/licenses/by-nc-nd/3.0/>. cerebral cortex, has been actively studied recently.

Action observation training is a training method that brings about improvements in actual skills through the activation of the brain region obtained by observing, imitating, and practicing others' actions ${ }^{8}$. The neurologic mechanism of this action observation training is based on the discovery of the mirror neuron system, in which regions similar to the premotor area and the parietal lobe in the brain region are known to be activated not only when actions are conducted firsthand, but also when the same actions are observed while they are being conducted ${ }^{9}$. While observing the behaviors of others, the nerves in the observer's brain involved in such behaviors are activated. Based on this, clinical studies are being continuously conducted in the area of nerve rehabilitation. In one study conducted with healthy adults based on this mirror neuron system, action observation training groups that observed actual actions and repeatedly practiced the same physical actions as they had observed actions showed significantly larger training effects than groups that repeated simple actions without observing actions ${ }^{10)}$. These results suggest that action observation training can be a more useful intervention method than task-oriented training for improving the motor skills of patients with impaired motor skills due to damage to the brain region such as that resulting from a stroke ${ }^{11}$. Recently, evidence has been presented supporting the effects of action observation training in the process of relearning functional tasks after a stroke ${ }^{12)}$, and proposals indicating that larger improvements in skills can be expected if action observation training is applied to patients in combination with the training currently used in interventions for rehabilitation have been raised ${ }^{13,14)}$. 
However, although many studies on action observation training have been conducted recently, most of them have been related to upper extremity functions, and studies that have examined the effects of action observation training on balance and walking ability, which are basic elements for actual independent life, are quite insufficient. Therefore, this study intended to apply action observation training to stroke patients to examine the effects of action observation training on balance and walking ability of patients in order to help in applying action observation training as a method of rehabilitation training.

\section{SUBJECTS AND METHODS}

The subjects of this study were 40 patients diagnosed with hemiplegia resulting from a stroke by a rehabilitation doctor practicing at N Hospital located in Daegu, South Korea. They were randomly and equally assigned to a action observational gait training group (AOGT: 10 males, 10 females) and a general gait training group (GGT: 11 males, 9 females). The mean \pm SD age, height, and weight of the AOGT were $51.15 \pm 14.81$ years, $163.45 \pm 8.53 \mathrm{~cm}$, and $64.1 \pm 13.1 \mathrm{~kg}$, respectively. Eleven of the patients had right hemiplegia, and nine had left hemiplegia. The onset period was $14.8 \pm 6.1$ months. The mean $\pm \mathrm{SD}$ age, height, and weight of the GGT were $48.65 \pm 12.81$ years, $165.9 \pm 8.6 \mathrm{~cm}$, and $63.80 \pm 9.26 \mathrm{~kg}$, respectively. Nine of the patients had right hemiplegia, and 11 had left hemiplegia. The onset period was $13.4 \pm 8.2$ months.

The inclusion criteria were as follows: no visual field defect, no abnormality in the vestibular organs, no orthopedic disease, an unrestricted range of motion, an ability to understand and perform the exercise as instructed by the researcher, and a score of 24 or higher on the Mini-Mental State Examination-Korean version. All of the subjects understood the purpose of this study and provided their written informed consent prior to their participation in the study in accordance with the ethical standards of the Declaration of Helsinki.

Prior to each training, the participants in both groups received 30 minutes of general physical therapy that consisted of a joint exercise, muscle strengthening exercise, and a stretching exercise.

The action observational gait training group watched a video that consisted of three-minute videos each for walking on a flat land, on a slope, and on steps, all executed by a healthy person, and took a one-minute break afterwards. Then, the group went through walking training for five minutes each on the same flat land, slope, and steps as in the video. Between each set of training, they took a 1-2 minute break, and in total, the walking training took 20 minutes. The general gait training group watched a video that showed images of nature unrelated with walking for the same amount of time, and went through the same walking training with the experimental group for 20 minutes. The experiment was conducted over the course of 30 minutes each time, five times per week, for a total of eight weeks. In the present study, a balance ability measuring and training system (analysis system using biofeedback, AP1153 BioRescue, France) was used to measure the balance ability of the subjects. The balance ability measuring and training system is suitable for static and dynamic measurement of the balance ability of patients, general people, and athletes. This system can be used to observe the moving path line of the center of pressure during certain movements to determine the length $(\mathrm{mm})$ of the moving path line, the average speed of the movements $(\mathrm{cm} / \mathrm{s})$, and the area of the movements. In the present study, the patient was instructed to spread his/her legs to around $30^{\circ}$ in an upright standing position and look forward. The measuring method was explained via a monitor and demonstrated before measurement.

The patient was instructed to keep his/her balance for one minute while looking forward. The distribution of weight bearing on the paretic and nonparetic sides, the total distance of movements of the center point of the body, and the area of the movements were then measured. To compare the patient's static balance, the limit of stability in a static standing position within which the patient could maximally move his/ her center of gravity while keeping balance in the direction instructed by the monitor using the ankle strategy in an upright standing position was measured. For the measurement of gait ability, we used the Timed Up and Go Test and the 10 Meter Walking Test.

The experimental results were statistically analyzed using SPSS 20.0 KO (IBM, Armomk, NY, USA). After the general characteristics of the subjects were determined, a paired t-test was used to measure the sway area, sway speed, and limit of stability in a static standing position. Tests were done before and after the intervention for each group. The significance of the differences between the two groups was investigated using an independent t-test. Statistical significance was accepted for values of $\mathrm{p}<0.05$.

\section{RESULTS}

With regard to changes in the sway area, sway speed, and limit of stability, both groups showed significant decreases after the intervention $(\mathrm{p}<0.05)$. There were significant differences in the sway speed and total length of the limit of stability between the two groups after the experiment $(p<0.05)$, but there were no significant differences in the sway area between the two groups after the experiment $(\mathrm{p}>0.05)$ (Table 1).

With regard to changes in the Timed Up and Go Test and the 10 Meter Walking Test, the both groups showed significant improvements after the intervention $(p<0.05)$. There were significant differences in the Timed Up and Go Test and the 10 Meter Walking Test between the two groups after the experiment $(\mathrm{p}<0.05)$ (Table 2$)$.

\section{DISCUSSION}

Improvements in the dynamic balance and walking ability of chronic stroke patients is considered an important element for improving their quality of life through their participation in social life and daily life and for increasing their social interactions $^{15}$.

The aim of this study was to examine the effects of action observation training on balance and walking in stroke patients. According to the results, after gait training for eight 
Table 1. Comparison of the static balance on standing position between the AOGT group and the GGT group (mean $\pm \mathrm{SD})$

\begin{tabular}{lcccc}
\hline & AOGT & \multicolumn{3}{c}{ GGT } \\
\cline { 2 - 5 } & Pretest & Posttest & Pretest & Posttest \\
\hline SA $\left(\mathrm{mm}^{2}\right)$ & $437.9 \pm 381.2$ & $167.5 \pm 166.2^{*}$ & $349.7 \pm 407.6$ & $349.7 \pm 407.6^{*}$ \\
SS $(\mathrm{mm} / \mathrm{s})$ & $0.8 \pm 0.3$ & $0.5 \pm 0.2^{*^{a}}$ & $0.7 \pm 0.2$ & $0.6 \pm 0.2^{*}$ \\
LOS $\left(\mathrm{mm}^{2}\right)$ & $4,235.5 \pm 4,644.7$ & $7,064.3 \pm 5,014.4^{* \mathrm{a}}$ & $2,361.1 \pm 2,299.5$ & $3,002.1 \pm 2,629.2^{*}$ \\
\hline
\end{tabular}

*Significant difference compared with before therapy at $<0.05$. ${ }^{a}$ significant difference in gains between the two groups at $<0.05$. AOGT: Action observation gait training group, GGT: General gait training group, SA: Sway Area, SS: Sway Speed, LOS: Limit of stability

Table 2. Comparison of AOGT and GGT groups' gait ability (mean \pm SD)

\begin{tabular}{lcccc}
\hline & \multicolumn{3}{c}{ AOGT } & \multicolumn{2}{c}{ GGT } \\
\cline { 2 - 5 } & Pretest & Posttest & Pretest & Posttest \\
\hline TUG (sec) & $20.0 \pm 3.5$ & $15.7 \pm 3.3^{*_{\mathrm{a}}}$ & $20.5 \pm 3.5$ & $17.7 \pm 3.2^{*}$ \\
10-M WT (sec) & $19.1 \pm 1.7$ & $14.6 \pm 2.3^{* \mathrm{a}}$ & $18.6 \pm 2.8$ & $15.5 \pm 2.3^{*}$ \\
\hline
\end{tabular}

${ }^{*}$ Significant difference compared with before therapy at $<0.05 .{ }^{\text {a }}$ significant difference in gains between the two groups at $<0.05$. AOGT: Action observation gait training group, GGT: General gait training group, TUG: Timed up and go test, 10-MWT: 10-meter walk test

weeks including walking action observation, the center of gravity sway areas, sway speeds, and movement distance of their stability limit in standing positions of the subjects were significantly improved, and significant improvement was also shown in the Timed Up and Go Test and the 10 Meter Walking Test after training. In comparison with the GGT group, statistically significant improvements were shown in all measured variables except for sway areas.

In a study conducted by Maloui et al. ${ }^{16}$ ) in which 12 stroke patients underwent physical training combined with imaginary training, the researchers reported that the weight bearing ratio of the paretic lower extremity increased after the intervention, and this effect was maintained even after 24 hours; in a study conducted by Choi and Park ${ }^{17)}$, the time spent on weight-shifting tasks, the movement distance of the center of pressure, and the Timed Up and Go Test scored decreased significantly in the group that underwent actual training combined with motor imagery. Dunsky et al. ${ }^{18)}$ reported improved walking functions with increases in walking speed, paretic side stride length, cadence, and single-support time and decreases in double-support time.

As with the abovementioned previous studies, in this study too, static and dynamic balance elements and walking elements were improved after patients underwent action observation training. Based on a previous study indicating that the same motor projection area is activated when observing actions as when actually performing the actions ${ }^{9}$, action observation is considered to have activated the neural networks in their cerebrum and cerebellum of the stroke patients, leading to the reorganization of their brain and bring about improvements in skills ${ }^{18)}$. This fact shows that action observation training can affect improvements in balance and walking ability. However, this study has limitations in that it could not be compared with other studies because few studies have been conducted in which action observation training has been implemented in stroke patients to examine changes in balance and walking ability, the long-term effects of action observation training could not be judged through this study, and the persistence of the effects are unknown, as the patients were not followed up.

With the indicators measured using the elements of walking in this study, only functional walking performance improvements could be judged. Hereafter, not only evaluation of the effects of action observation training on the functional aspects of walking but also temporal/spatial and kinematic evaluation and evaluation of effects in terms of neurology should be conducted more, and more diverse action observation programs and tasks should be implemented.

Based on the results of this study, action observation training is considered an effective method that can be used clinically because it can be easily implemented by stroke patients who are motivated with respect to rehabilitation, since it is composed of processes consisting of observation of movement task videos and practice of observed tasks; it can also be useful in terms of economic efficiency, as patients can continue training using videos even after they leave the hospital. Action observation training may be effective for improving skills if it is utilized in combination with traditional treatment methods as a preparation process before training in stroke rehabilitation. However, this training could only be continued when patients had high levels of concentration and were strongly motivated with respect to rehabilitation while they were observing actions. Moreover, the video contents were insufficient. Hereafter, studies to provide standardized frameworks should be continued so that clinical therapists can more clearly design action observation training program.

\section{REFERENCES}

1) Lee JH, Park SW, Kim DA, et al.: Gait analysis using accelerometer in stroke patients. J Korean Acad Rehabil Med, 2004, 28: 488-493. 
2) Gracanin F, Prevec F, Trontelj I: Evaluation of use of functional electronic peroneal brace in hemiparetic patients, Belgrade: Yugoslav Committee for Electronics and Automation, 1967, 198-205.

3) Flansbjer UB, Lexell J, Brogårdh C: Long-term benefits of progressive resistance training in chronic stroke: a 4-year follow-up. J Rehabil Med 2012, 44: 218-221. [Medline] [CrossRef]

4) Grealy MA, Johnson DA, Rushton SK: Improving cognitive function after brain injury: the use of exercise and virtual reality. Arch Phys Med Rehabil, 1999, 80: 661-667. [Medline] [CrossRef]

5) Lee J, Seo K: The effects of stair walking training on the balance ability of chronic stroke patients. J Phys Ther Sci, 2014, 26: 517-520. [Medline] [CrossRef]

6) Ada L, Dean CM, Vargas J, et al.: Mechanically assisted walking with body weight support results in more independent walking than assisted overground walking in non-ambulatory patients early after stroke: a systematic review. J Physiother, 2010, 56: 153-161. [Medline] [CrossRef]

7) Cicinelli $\mathrm{P}$, Marconi B, Zaccagnini $\mathrm{M}$, et al.: Imagery-induced cortica excitability changes in stroke: a transcranial magnetic stimulation study. Cereb Cortex, 2006, 16: 247-253. [Medline] [CrossRef]

8) Jeannerod M: The hand and the object: the role of posterior parietal cortex in forming motor representations. Can J Physiol Pharmacol, 1994, 72 535-541. [Medline] [CrossRef]

9) Grèzes J, Decety J: Functional anatomy of execution, mental simulation, observation, and verb generation of actions: a meta-analysis. Hum Brain Mapp, 2001, 12: 1-19. [Medline] [CrossRef]

10) Celnik P, Stefan K, Hummel F, et al.: Encoding a motor memory in the older adult by action observation. Neuroimage, 2006, 29: 677-684. [Med- line] [CrossRef]

11) Buccino G, Solodkin A, Small SL: Functions of the mirror neuron system implications for neurorehabilitation. Cogn Behav Neurol, 2006, 19: 55-63. [Medline] [CrossRef]

12) Ertelt D, Small S, Solodkin A, et al.: Action observation has a positive impact on rehabilitation of motor deficits after stroke. Neuroimage, 2007, 36: T164-T173. [Medline] [CrossRef]

13) Franceschini M, Agosti M, Cantagallo A, et al.: Mirror neurons: action observation treatment as a tool in stroke rehabilitation. Eur J Phys Rehabil Med, 2010, 46: 517-523. [Medline]

14) Lee D, Roh H, Park J, et al.: Drinking behavior training for stroke patients using action observation and practice of upper limb function. J Phys Ther Sci, 2013, 25: 611-614. [Medline] [CrossRef]

15) Lord SE, Rochester L, Weatherall M, et al.: The effect of environment and task on gait parameters after stroke: a randomized comparison of measurement conditions. Arch Phys Med Rehabil, 2006, 87: 967-973. [Medline] [CrossRef]

16) Malouin F, Belleville S, Richards CL, et al.: Working memory and mental practice outcomes after stroke. Arch Phys Med Rehabil, 2004, 85: 177-183. [Medline] [CrossRef]

17) Choi JH, Park SH: The effect of weight shifting and motor imagery training on the ability of weight shifting task and functional activity in stroke patients. J Spec Educ Rehabil Sci, 2009, 48: 169-182.

18) Dunsky A, Dickstein R, Marcovitz E, et al.: Home-based motor imagery training for gait rehabilitation of people with chronic poststroke hemiparesis. Arch Phys Med Rehabil, 2008, 89: 1580-1588. [Medline] [CrossRef] 